\title{
Differences in the efficacy and safety of eribulin in patients with soft tissue sarcoma by histological subtype and treatment line
}

\author{
KENJI NAKANO ${ }^{1}$, KEIKO HAYAKAWA ${ }^{2}$, YUKI FUNAUCHI ${ }^{2}$, TAISUKE TANIZAWA ${ }^{2}$, \\ KEISUKE AE ${ }^{2}$, SEIICHI MATSUMOTO ${ }^{2}$, JUNICHI TOMOMATSU ${ }^{1}$, MAKIKO ONO ${ }^{1}$, \\ SHINICHIRO TAIRA ${ }^{1}$, MASATOSHI NISHIZAWA ${ }^{1}$, XIAOFEI WANG ${ }^{1}$, AKIHIRO OHMOTO $^{1}$, \\ YASUYOSHI SATO ${ }^{1}$, NAOKI FUKUDA ${ }^{1}$, TETSUYA URASAKI ${ }^{1}$ and SHUNJI TAKAHASHI ${ }^{1}$ \\ Departments of ${ }^{1}$ Medical Oncology and ${ }^{2}$ Orthopedic Oncology, \\ Cancer Institute Hospital of The Japanese Foundation for Cancer Research, Tokyo 135-8550, Japan
}

Received May 14, 2020; Accepted November 6, 2020

DOI: $10.3892 / \mathrm{mco} .2020 .2175$

\begin{abstract}
Clinical evidence regarding eribulin treatment for patients with soft tissue sarcoma (STS) is limited to those with L-sarcoma (leiomyosarcoma and liposarcoma) who have completed at least two chemotherapies. Whether histological subtypes and treatment lines affect the efficacy and safety of eribulin for patients with STS has yet to be elucidated. The current study retrospectively reviewed patients with STS receiving eribulin at the Cancer Institute Hospital of JFCR and evaluated the prognostic factors affecting its efficacy and safety by histological diagnoses and treatment lines. A total of 41 patients with STS, including 26 with L-sarcoma, underwent eribulin treatment. Additionally, a total of and 14 patients, including 12 with L-sarcoma, received eribulin as a second-line treatment. The results revealed that patients with L-sarcoma demonstrated longer progression-free survival (PFS) rates compared with patients without L-sarcoma (4.5 vs. 2.3 months; $\mathrm{P}=0.005)$. Furthermore, differences in treatment line significantly affected PFS (4.5 months in second-line treatment vs. 2.4 months in later lines; $\mathrm{P}=0.037$ ). A high number of patients with L-sarcoma received eribulin as a second-line treatment. Regarding safety, several adverse events were reported, such as neutropenia, which were more frequently observed in patients with L-sarcoma or other patients receiving eribulin as a second-line treatment. However, most adverse events were tolerable. The clinical efficacy of eribulin was increased in patients with L-sarcoma, which was similar to previous clinical trials. However, treatment lines could also affect its efficacy. When evaluating the clinical value of eribulin to STS, it is important to consider treatment lines.
\end{abstract}

Correspondence to: Dr Kenji Nakano, Department of Medical Oncology, Cancer Institute Hospital of The Japanese Foundation for Cancer Research, Ariake 3-8-31, Tokyo 135-8550, Japan

E-mail: kenji.nakano@jfcr.or.jp

Key words: soft tissue sarcoma, chemotherapy, leiomyosarcoma, liposarcoma, eribulin

\section{Introduction}

The prognoses of patients with recurrent and/or metastatic soft tissue sarcoma (STS) remain poor, with median survival according to recent data limited to 14-20 months (1). Standard chemotherapy as a first-line treatment for STS is generally a doxorubicin-based regimen in most histological subtypes, but there are some systemic treatment options, including eribulin, available to STS patients refractory and/or intolerant to doxorubicin (2). The clinical evidence to date regarding eribulin in STS patients in a phase III trial has, however, been limited to L-sarcoma patients, i.e., those with leiomyosarcoma or liposarcoma, who had received at least two previous systemic chemotherapy treatments, including anthracyclines (3). As a result, the approval of eribulin for STS in the U.S. and Europe has been limited to patients with specific histological subtypes and treatment histories (4).

In Japan, eribulin has been approved for recurrent and/or metastatic STS patients regardless of histological subtype or treatment history, although its indication as a first-line therapy is not recommended. However, there is still insufficient clinical data about whether and how much differences in histological subtype and treatment line affect its efficacy. Differences in the efficacy of eribulin for STS in patients with different histological subtypes have been reported in previous phase II trials $(5,6)$ but, to the best of our knowledge, it has not yet been examined in different treatment lines or with respect to the number of previous systemic chemotherapies. The purpose of the present study, therefore, was to evaluate certain clinical factors that might affect the efficacy and safety of eribulin in STS patients.

\section{Materials and methods}

Patient data. We retrospectively reviewed the clinical records of recurrent and/or metastatic STS patients who underwent eribulin treatment and evaluated its efficacy and safety, analyzing prognostic factors by histological subtype and treatment line, which were defined as the number of chemotherapy regimens in a recurrent/metastatic setting. The patients who were diagnosed as STS by histological pathology, either 
obtained from biopsy or surgical resection were included in the analysis, and patients who were intended to receive eribulin but actually never received by disease progression, patients refusal or other reasons were excluded. This retrospective study was approved by the Institutional Review Board of Cancer Institute Hospital of Japanese Foundation for Cancer Research and written informed consent was obtained from each patient.

Statistical analysis. The patients' objective response and disease progression were evaluated using the Response Evaluation Criteria In Solid Tumours (RECIST), version 1.1. Adverse events were documented based on the Common Terminology Criteria for Adverse Events (CTCAE), version 5.0. To evaluate prognoses, both overall survival (OS) and progression-free survival (PFS) were estimated by the Kaplan-Meier method. The log-rank test was used to compare prognoses. The Chi-square test was used to compare the differences in the characteristics, chemotherapy treatment details and adverse events. The SPSS software ver. 25.0 was used for all statistical analyses, and $\mathrm{P}<0.05$ was considered to indicate a statistically significant difference.

\section{Results}

Patient data. A total of 41 STS patients were treated with eribulin between March 2016 and December 2018, 26 of whom had L-sarcoma (12 leiomyosarcoma and 14 liposarcoma). The histological subtypes of non-L-sarcoma patients were as follows: 8 undifferentiated pleomorphic sarcoma, 2 synovial sarcoma, 1 clear-cell sarcoma, 1 endometrial stromal sarcoma, 1 myxoid chondrosarcoma, 1 malignant peripheral nerve sheath tumor, and 1 solitary fibrous tumor. The characteristics of the enrolled patients are summarized in Table I. There were no significant differences in the patients' gender, age or primary lesion between the L-sarcoma and non-L-sarcoma groups, but there were differences in the number of previous chemotherapies: Nearly half of L-sarcoma patients received eribulin as a second-line therapy, while most non-L-sarcoma patients had received two or more chemotherapies in a recurrent/metastatic setting. In L-sarcoma patients, higher rates of liposarcoma patients (11 of 14 patients; 78.6\%) than leiomyosarcoma patients ( 1 of $12 ; 8.3 \%$ ) received eribulin as a second-line therapy. All patients received doxorubicin as a perioperative or earlier-line chemotherapy regardless of histological subtype (Table II). History of radiation therapy was observed in $21(51.2 \%)$ patients, including 8 primary lesions (4 retroperitoneal/intra-abdomen, 2 soft tissue of trunk, 1 thoracic lesion, and 1 head and neck) and 13 metastatic lesions ( 7 bone metastases, 5 lung/thoracic metastases and 1 soft tissue or trunk).

Statistical analysis. A comparison of patients' prognoses by histological subtype is shown in Fig. 1. The median PFS in L-sarcoma patients was statistically longer than in non-L-sarcoma patients (4.5 months vs. 2.3 months, $\mathrm{P}=0.005$; Fig. 1A), and the median OS also tended to be longer in L-sarcoma patients though statistical significance was not reached (20.0 months vs. 12.5 months, $\mathrm{P}=0.065$; Fig. 1C). In L-sarcomas, there might be differences in prognoses between leiomyosarcoma and liposarcoma. The PFS of liposarcoma patients was statistically longer than that of leiomyosarcoma patients (8.0 vs. 2.2 months, $\mathrm{P}=0.019$; Fig. 1B), but in terms of OS, there were no significant differences between liposarcoma and leiomyosarcoma (14.7 vs. 20.0 months, $\mathrm{P}=0.658$ ).

A comparison of patients' prognoses by treatment line is shown in Fig. 2. Patients receiving eribulin as a second-line treatment tended to have a better prognosis, though statistical significance was observed only in the PFS of all patients. Objective responses were observed in three liposarcoma patients $(7.3 \%)$.

History of radiation therapy did not affect the effectiveness of eribulin; the PFS of patients with and without history of radiation therapy were both 2.7 months $(\mathrm{P}=0.125)$ and the OS were 13.8 and 14.7 months $(\mathrm{P}=0.282)$.

Adverse events associated with eribulin are shown in Table III. Myelosuppresion, especially neutropenia and anemia, was highly observed. Non-hematological adverse events such as gastrointestinal events such as anorexia and nausea, as well as increased transaminase and neuropathy, were observed in relatively high incidence, but severe non-hematological adverse events were rare. Regarding histological subtype, leukocytopenia, neutropenia, thrombocytopenia, constipation and neuropathy were observed in $20 \%$ or more of L-sarcoma patients; regarding treatment line, neutropenia was observed in $20 \%$ or more of patients with second-line treatment. No patients terminated eribulin treatment due to adverse events, and there were no lethal adverse events.

\section{Discussion}

Eribulin is an antitumor-drug derived from the marine sponge Halichondria okadai, which acts as a microtubule dynamics inhibitor. The clinical benefit of eribulin to STS was confirmed by phase III trial (E7389-G00-309) in which eribulin showed significant OS prolongation compared to dacarbazine (3). This phase III trial, however, was designed to enroll only patients with L-sarcoma who had received at least two previous systemic chemotherapy treatments, including anthracycline. Thus, high-level clinical evidence regarding eribulin for STS is limited to L-sarcoma as a 3rd- or later-line therapy.

Differences in the efficacy of eribulin by histological subtype have been evaluated in some analyses. One phase II trial incorporating STS patients with various STS histological subtypes suggested that the survival benefits of eribulin for STS was greater in L-sarcoma patients (5), and was the basis of the inclusion criteria for the 309 phase III trial. In Japan, a similar tendency has been observed in both prospective and retrospective data: The prognoses of L-sarcoma patients receiving eribulin tends to be longer than those of non-L-sarcoma patients (6-8). Our retrospective analysis is also consistent with previous clinical data (2), with differences in the PFS of leiomyosarcoma and liposarcoma patients (Fig. 1B). In a subanalysis of the 309 phase III trial, in terms of hazard ratio to control arm, the OS benefit of eribulin was not relevant in leiomyosarcoma compared to liposarcoma $(9,10)$, and in the U.S. and Europe, leiomyosarcoma is excluded from eribulin indications (4). The OS, PFS and response rates in the 309 phase III trial, however, were not so change between leiomyosarcoma and liposarcoma. The differences in PFS between leiomyosarcoma and liposarcoma in our analysis 
Table I. Characteristics of the patients enrolled in the present study.

\begin{tabular}{|c|c|c|c|c|}
\hline Characteristics & All patients, n (\%) & L-sarcoma, n (\%) & Non-L-sarcoma, n (\%) & P-value (Two-tailed $\chi^{2}$ ) \\
\hline Sex & & & & 0.097 \\
\hline Male & $16(39.0)$ & $13(50.0)$ & $3(20.0)$ & \\
\hline Female & $25(61.0)$ & $13(50.0)$ & $12(80.0)$ & \\
\hline \multicolumn{5}{|l|}{ Age, years } \\
\hline Median & 61 & 64 & 54 & \\
\hline Range & $23-75$ & $44-74$ & $23-75$ & \\
\hline Age $\geq 60$ years & $24(58.5)$ & $17(65.4)$ & - & 0.200 \\
\hline Primary lesion & & & & 0.453 \\
\hline Extremities & $10(24.4)$ & $5(19.2)$ & $5(33.3)$ & \\
\hline Non-extremities & $31(75.6)$ & $21(80.8)$ & $10(66.7)$ & \\
\hline \multicolumn{5}{|l|}{ Treatment history } \\
\hline Surgery & $36(87.8)$ & $23(88.5)$ & $13(86.7)$ & $>0.999$ \\
\hline Radiation & $21(51.2)$ & $12(46.2)$ & $9(60.0)$ & 0.520 \\
\hline Perioperative chemotherapy & $13(31.7)$ & $8(30.8)$ & $5(33.3)$ & $>0.999$ \\
\hline Treatment lines & & & & 0.044 \\
\hline Second line & $14(34.1)$ & $12(46.2)$ & $2(13.3)$ & \\
\hline Later lines & 27 (65.9) & $14(53.8)$ & $13(86.7)$ & \\
\hline Total & 41 & 26 & 15 & \\
\hline
\end{tabular}

Table II. Details of the antitumor drugs used as previous chemotherapy, including preoperative treatments.

\begin{tabular}{|c|c|c|c|c|c|}
\hline \multirow[b]{2}{*}{ Antitumor drug } & \multicolumn{2}{|c|}{ All patients } & \multicolumn{2}{|c|}{ L-sarcoma patients } & \multirow[b]{2}{*}{ P-value (Two-tailed $\chi^{2}$ ) } \\
\hline & 2nd line, n (\%) & Later lines, n (\%) & 2nd line, n (\%) & Later lines, $\mathrm{n}(\%)$ & \\
\hline Doxorubicin & $14(100.0)$ & $26(96.3)$ & $12(100.0)$ & $14(100.0)$ & 0.366 \\
\hline Ifosfamide & $11(78.6)$ & $17(63.0)$ & $2(16.7)$ & $9(64.3)$ & 0.341 \\
\hline Gemcitabine & $1(7.1)$ & $17(63.0)$ & $0(0.0)$ & $11(78.6)$ & $>0.999$ \\
\hline Docetaxel & $1(7.1)$ & $13(48.1)$ & $0(0.0)$ & $9(64.3)$ & $>0.999$ \\
\hline Pazopanib & $0(0.0)$ & $20(74.1)$ & $0(0.0)$ & $10(71.4)$ & 0.111 \\
\hline Trabectedin & $2(14.2)$ & $5(18.5)$ & $2(16.7)$ & $2(14.3)$ & 0.693 \\
\hline Other & $2(14.2)$ & $9(33.3)$ & $1(8.3)$ & $7(50.0)$ & $>0.999$ \\
\hline Total & 14 & 27 & 12 & 14 & - \\
\hline
\end{tabular}

${ }^{\mathrm{a} A l l}$ patients were compared with patients with L-sarcoma.

might have been influenced by another confounding factor; one candidate is the difference in treatment line.

In the 309 phase III trial, a treatment history of two or more chemotherapies was the indication for enrollment, and there were no apparent differences in treatment line between the eribulin arm and the control arm, nor between leiomyosarcoma and liposarcoma in the eribulin arm $(9,10)$. In our retrospective analysis, liposarcoma patients tended to receive eribulin as a second-line therapy at high rates, which might have led to the differences in PFS between leiomyosarcoma and liposarcoma patients, arising from the fact that that there were other treatment options considered to be more effective in leiomyosarcoma patients than in liposarcoma patients, such as gemcitabine-based therapy and pazopanib $(11,12)$. In fact, our retrospective analysis showed no changes in OS between leiomyosarcoma and liposarcoma, which might be a result of differences in treatment options other than eribulin.

Regarding safety, L-sarcoma patients with or without a second line, who showed longer PFS, tended to have had more cumulative doses of eribulin, so some adverse events related to cumulative exposure to eribulin, such as myelosuppression and peripheral neuropathy, were observed in L-sarcoma patients and/or second-line patients (Table III), but most of these events were considered tolerable.

Recently, personalized therapies for STS have emerged, and biomarkers and/or patients' factors affecting the efficacy of eribulin have been evaluated $(13,14)$. The histological subtype of L-sarcoma is now considered to be a positive 
A

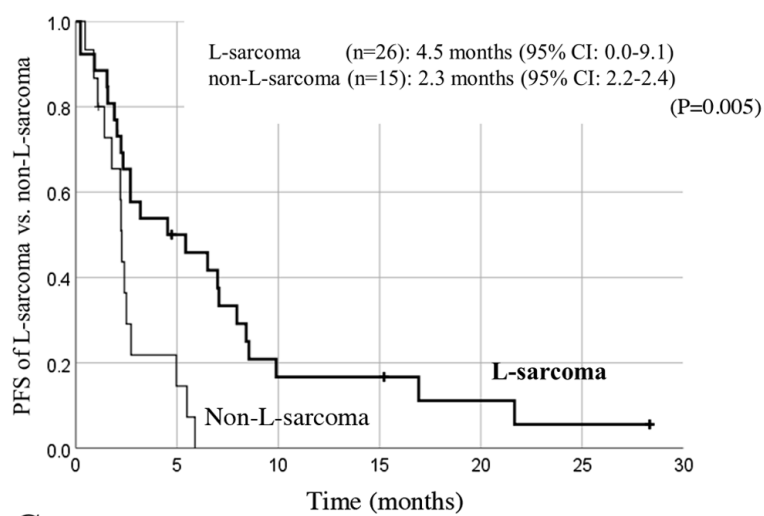

C

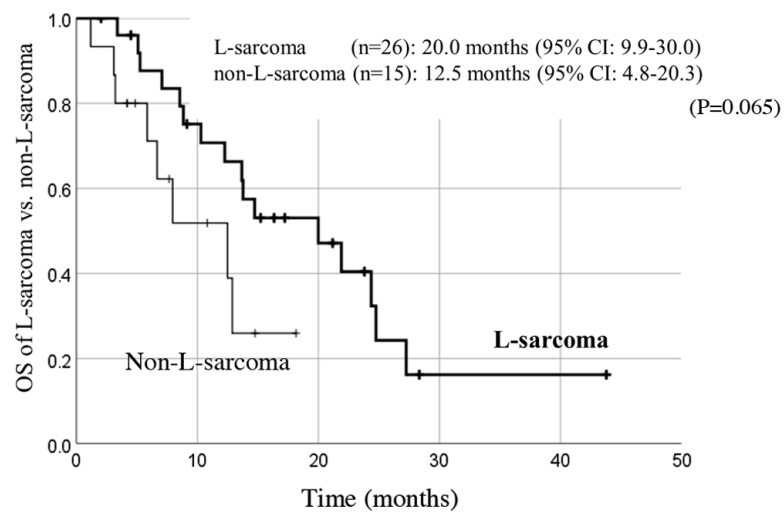

B

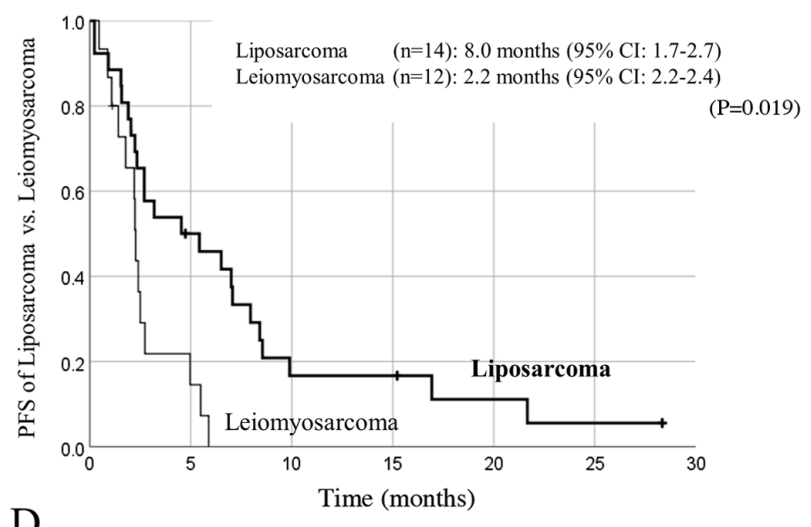

D

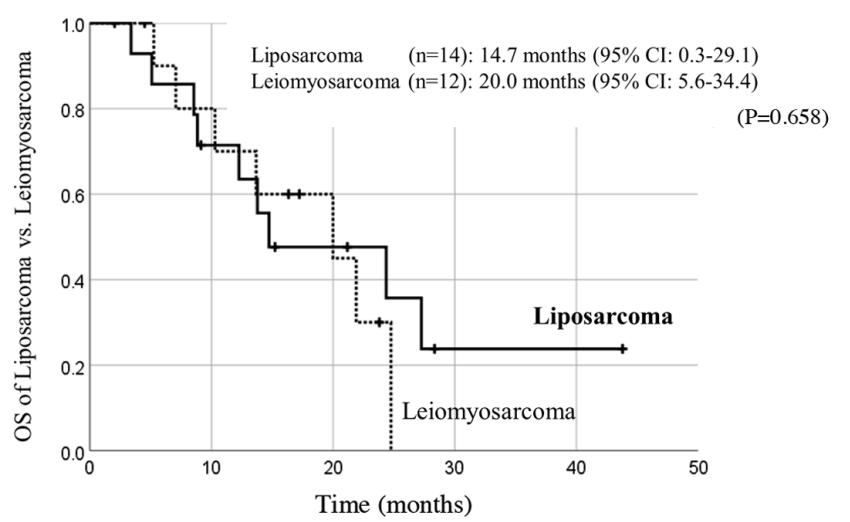

Figure 1. (A and B) PFS and (C and D) OS of patients with soft tissue sarcoma treated with eribulin by histological subtype. PFS, progression-free survival; OS, overall survival; CI, confidence interval.

A

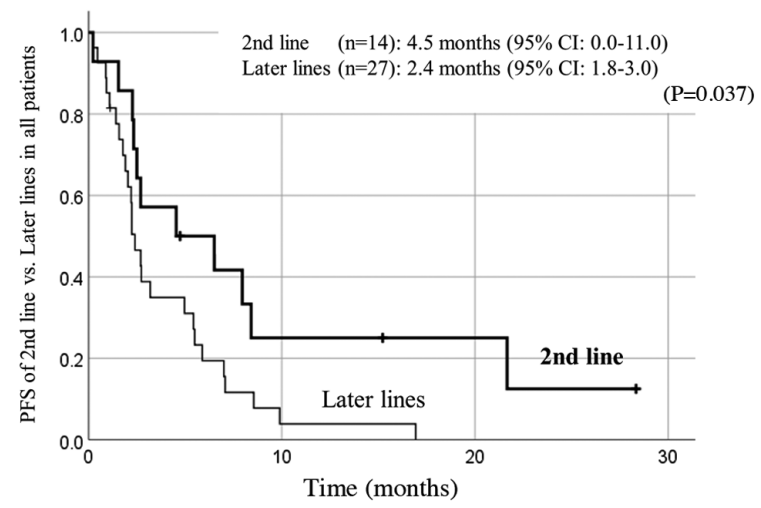

$\mathrm{C}$

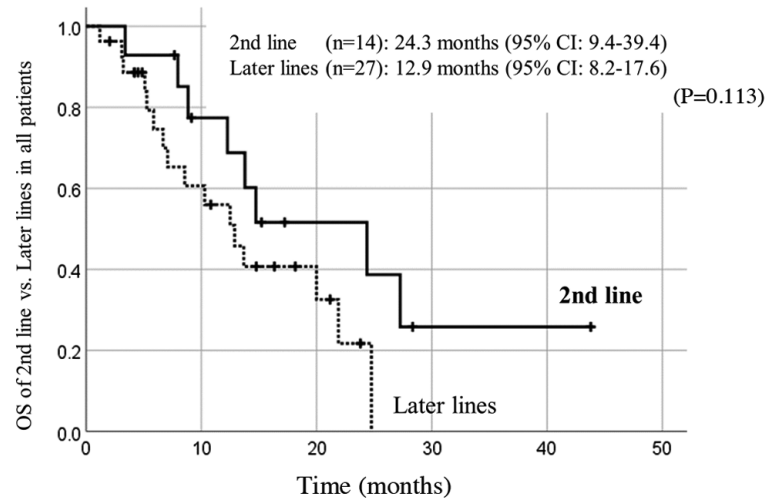

B

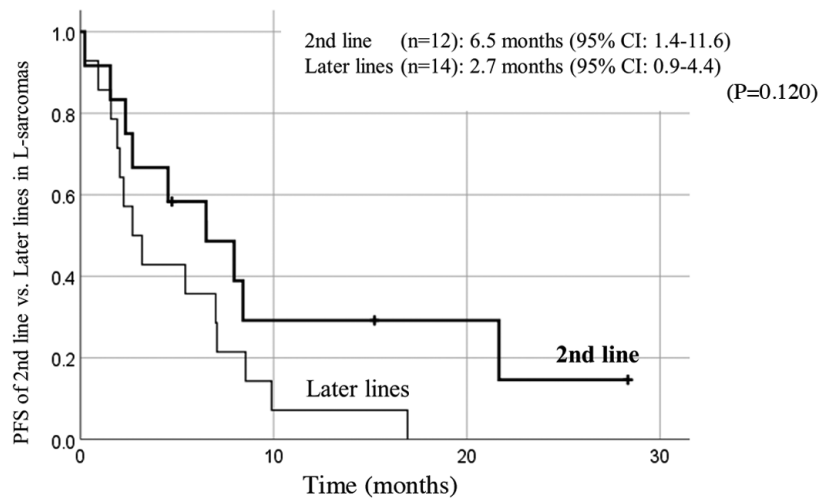

D

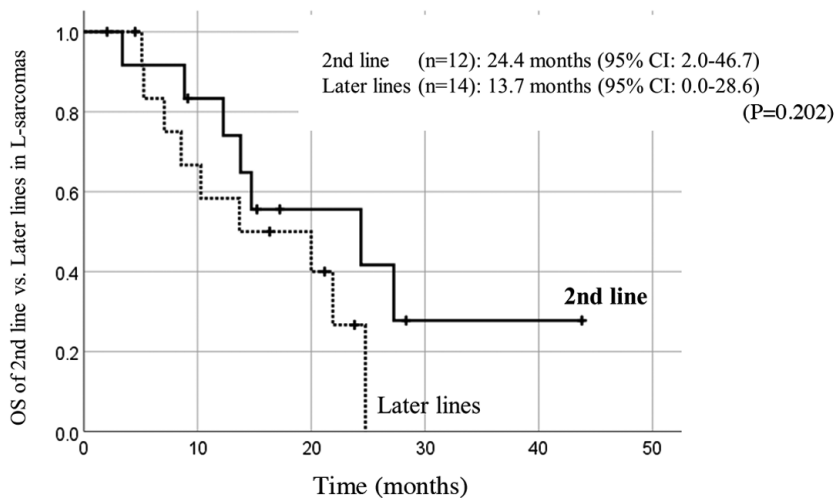

Figure 2. (A and B) PFS (C and D) OS of patients with soft tissue sarcoma treated with eribulin by treatment line. PFS, progression-free survival; OS, overall survival; $\mathrm{CI}$, confidence interval. 


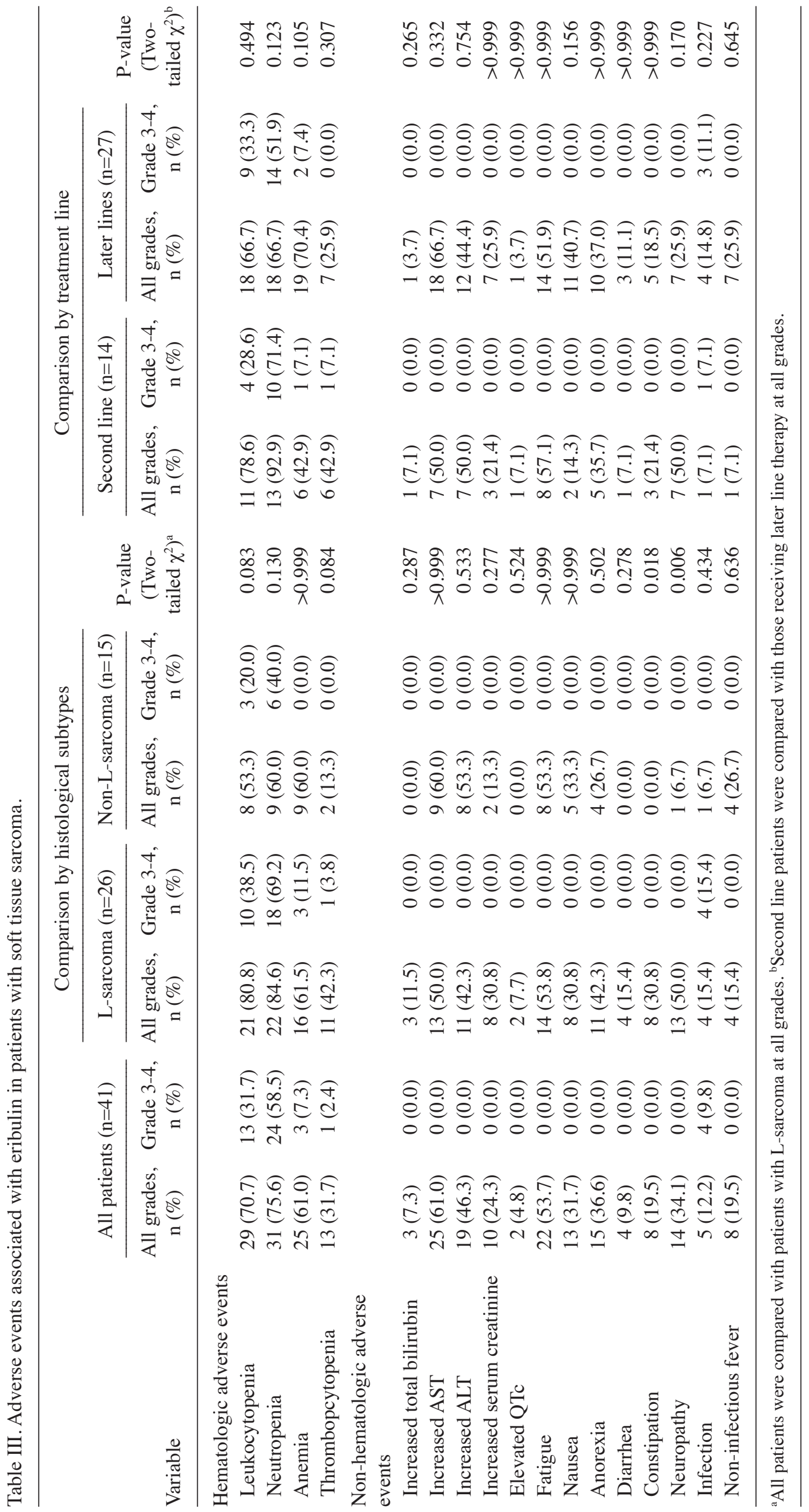


predictive factor of efficacy of eribulin, but there have been non-L-sarcoma patients whose clinical data indicate a benefit from eribulin treatment $(7,8)$. Treatment line, however, also affects the efficacy of eribulin and the patient's prognosis, so in considering new predictive markers of eribulin, the possibility of differences in treatment line should be considered.

\section{Acknowledgements}

Not applicable.

\section{Funding}

No funding was received.

\section{Availability of data and materials}

The datasets used and/or analyzed during the present study are available from the corresponding author on reasonable request.

\section{Authors' contributions}

$\mathrm{KN}$ designed the study and wrote the initial draft of the manuscript. KH, YF, TT, KA, SM, JT, MO, ST, MN, XW, AO, YS, NF and TU provided clinical treatment. ST supervised the manuscript and clinical treatment. All authors approved the final version of the manuscript.

\section{Ethics approval and consent to participate}

This retrospective study was approved by the institutional review board of Cancer Institute Hospital of Japanese Foundation for Cancer Research and written informed consent was obtained from each patient. Written informed consent was obtained from each participant.

\section{Patient consent for publication}

Patient consent for publication was obtained from each participant.

\section{Competing interests}

The authors declare that they have no competing interests.

\section{References}

1. DeVita VT Jr, Lawrence TS and Rosenberg SA: DeVita, Hellman, and Rosenberg's Cancer Principles \& Practice of Oncology 11th edition. Lippincott Williams \& Wilkins, Philadelphia, PA, 2018.

2. Kawai A, Yonemori K, Takahashi S, Araki N and Ueda T: Systemic therapy for soft tissue sarcoma: Proposals for the optimal use of pazopanib, trabectedin, and eribulin. Adv Ther 34: $1556-1571,2017$
3. Schöffski P, Chawla S, Maki RG, Italiano A, Gelderblom H, Choy E, Grignani G, Camargo V, Bauer S, Rha SY, et al: Eribulin versus dacarbazine in previously treated patients with advanced liposarcoma or leiomyosarcoma: A randomised, open-label, multicentre, phase 3 trial. Lancet 387: 1629-1637, 2016.

4. Osgood CL, Chuk MK, Theoret MR, Huang L, He K, Her L, Keegan P and Pazdur R: FDA approval summary: Eribulin for patients with unresectable or metastatic liposarcoma who have received a prior anthracycline-containing regimen. Clin Cancer Res 23: 6384-6389, 2017.

5. Schöffski P, Ray-Coquard IL, Cioffi A, Bui NB, Bauer S, Hartmann JT, Krarup-Hansen A, Grünwald V, Sciot R, Dumez H, et al: European Organisation for Research and Treatment of Cancer (EORTC) Soft Tissue and Bone Sarcoma Group (STBSG). Activity of eribulin mesylate in patients with soft-tissue sarcoma: A phase 2 study in four independent histological subtypes. Lancet Oncol 12: 1045-1052, 2011.

6. Kawai A, Araki N, Naito Y, Ozaki T, Sugiura H, Yazawa Y, Morioka H, Matsumine A, Saito K, Asami S and Isu K: Phase 2 study of eribulin in patients with previously treated advanced or metastatic soft tissue sarcoma. Jpn J Clin Oncol 47: 137-144, 2017.

7. Kobayashi E, Naito Y, Asano N, Maejima A, Endo M, Takahashi S, Megumi Y and Kawai A: Interim results of a real-world observational study of eribulin in soft tissue sarcoma including rare subtypes. Jpn J Clin Oncol 49: 938-946, 2019.

8. Nakamura T, Tsukushi S, Asanuma K, Katagiri H, Ikuta K, Nagano A, Kozawa E, Yamada S, Shido Y, Yamada K, et al: The clinical outcome of eribulin treatment in Japanese patients with advanced soft tissue sarcoma: A Tokai Musculoskeletal oncology consortium study. Clin Exp Metastasis 36: 343-350, 2019.

9. Blay JY, Schöffski P, Bauer S, Krarup-Hansen A, Benson C, D'Adamo DR, Jia Y and Maki RG: Eribulin versus dacarbazine in patients with leiomyosarcoma: Subgroup analysis from a phase 3, open-label, randomised study. Br J Cancer 120: 1026-1032, 2019

10. Demetri GD, Schöffski P, Grignani G, Blay JY, Maki RG, Van Tine BA, Alcindor T, Jones RL, D'Adamo DR, Guo M and Chawla S: Activity of eribulin in patients with advanced liposarcoma demonstrated in a subgroup analysis from a randomized phase III study of eribulin versus dacarbazine. J Clin Oncol 35: 3433-3439, 2017.

11. Hensley ML, Maki R, Venkatraman E, Geller G, Lovegren M, Aghajanian C, Sabbatini P, Tong W, Barakat R and Spriggs DR: Gemcitabine and docetaxel in patients with unresectable leiomyosarcoma: Results of a phase II trial. J Clin Oncol 20: 2824-2831, 2002

12. van der Graaf WT, Blay JY, Chawla SP, Kim DW, Bui-Nguyen B, Casali PG, Schöffski P, Aglietta M, Staddon AP, Beppu Y, et al: Pazopanib for metastatic soft-tissue sarcoma (PALETTE): A randomised, double-blind, placebo-controlled phase 3 trial. Lancet 379: 1879-1886, 2012.

13. Wiemer EAC, Wozniak A, Burger H, Smid M, Floris G, Nzokirantevye A, Sciot R, Sleijfer S and SchöffskiP: Identification of microRNA biomarkers for response of advanced soft tissue sarcomas to eribulin: Translational results of the EORTC 62052 trial. Eur J Cancer 75: 33-40, 2017.

14. Kobayashi H, Okuma T, Oka H, Okajima K, Ishibashi Y, Zhang L, Hirai T, Ohki T, Tsuda Y, Ikegami M, et al: Body composition as a predictor of toxicity after treatment with eribulin for advanced soft tissue sarcoma. Int J Clin Oncol 24: 437-444, 2019.

(i) (9) This work is licensed under a Creative Commons Attribution-NonCommercial-NoDerivatives 4.0 International (CC BY-NC-ND 4.0) License. 